\title{
EFECTIVIDAD EDUCATIVA DEL FACEBOOK PARA EL DESARROLLO DE LA COMPRENSIÓN LECTORA
}

\author{
Educational effectiveness of Facebook for development \\ of the reading understanding
}

Javier Enrique Bernier Yanes*

\section{RESUMEN}

El propósito de este estudio fue determinar la efectividad educativa del Facebook (FB) para el desarrollo de la comprensión lectora, utilizando como principales autores a Piscitelli, Adaime y Binder (2010), Gordillo y Flórez (2009), Pérez (2016), entre otros. La investigación corresponde a un estudio de caso de tipo descriptivo con diseño de campo y cuasi-experimental, cuyos resultados indican que para una muestra de 40 estudiantes divididos en dos grupos (A y B), hay clara evidencia de mejoras significativas en la comprensión lectora de los estudiantes que utilizaron el FB a diferencia de aquellos que emplearon el método de lectura tradicional, evidenciando la efectividad de educativa de esta red social para el desarrollo de habilidades lectoras. Estos resultados, sustentan la necesidad de implementar estrategias de enseñanzaaprendizaje basadas en la aplicación del FB para hacer la lectura más amena, motivadora e interactiva, de forma que facilite la comprensión e interpretación del texto escrito para la construcción de nuevos conocimientos

Palabras clave: Comprensión lectora, habilidades lectoras, Facebook.

RECIBIDO: Junio 2018

ACEPTADO: Enero 2019

\section{ABSTRACT}

The purpose of this study was to determine the educational effectiveness of Facebook (FB) for the development of reading understanding, using as main authors Piscitelli, Adaime and Binder (2010), Gordillo and Flórez (2009), Pérez (2016), among others.

\footnotetext{
* Magister en Informática Educativa. Especialista en Informática y Telemática. Licenciado en Educación Artística. Docente de tiempo completo de la Universidad Cooperativa de Colombia y catedrático de la Universidad del Magdalena. Santa Marta, Colombia. Correo electrónico: profesorbernier@hotmail.com
} 
The investigation corresponds to a case study descriptive with field design and quasiexperimental, whose results indicate that for a sample of 40 students divided into two groups (A and B), there is clear evidence of significant improvements in the reading comprehension of the students who used the FB as opposed to those who used the traditional reading method, evidencing the educational effectiveness of this social network for the development of reading skills. These results support the need to implement teaching-learning strategies based on the application of the FB to make reading more enjoyable, motivating and interactive, in a way that facilitates the understanding and interpretation of the written text for the construction of new knowledge.

Keywords: Reading understanding, reading skills, Facebook

\section{Introducción.}

En el contexto educativo actual la Web 2.0 ha generado un alto grado de interconectividad entre las personas, quienes comparten en el espacio virtual información, datos, archivos, gustos, afinidades a través de una amplia variedad de redes sociales como el Facebook, cuyo uso se ha diversificado y adaptado a nuevas modalidades de enseñanza-aprendizaje traspasando el ámbito de interacción social adjudicado por los usuarios desde su origen.

A estas instancias, la red social es entendida como "una arquitectura expresada en el código informático, integra una comunidad caracterizada por un ethos propio, escala de valores particular o un lenguaje que puede ser aprendido y entendido críticamente" (Piscitelli, Adaime y Binder, 2010, p.12). Por consiguiente, es un espacio virtual de trabajo que permite alcanzar una construcción colectiva que puede determinar diálogos e interacciones generadoras de experiencias diversas dentro de las cuales se incluye la comprensión lectora.

En este sentido, el FB facilita la lectura social a través del intercambio de opiniones entre los estudiantes y el docente, permite el acceso a la intertextualidad utilizada para promover la comprensión inferencial del texto escrito, la interpretación de discursos y relacionar fragmentos de obras con experiencias previas profundizando el análisis crítico-reflexivo del contenido. 
La lectura concebida como una fase de creación que aporta, enriquece y recrea el texto, es un proceso que le permite al lector construir significados de forma activa, aplicar estrategias efectivas de lectura y reflexionar sobre su propio proceso lector (Guthrie y Taboada, 2005). Leer es por lo tanto, dotar de significado todo aquello que se lee, es una interpretación comprensiva de lo que se descubre en la lectura a partir de la propia realidad del sujeto, de su cultura y del contexto donde la realiza. Así, se incorpora lo leído al propio esquema del lector, valorando reflexiva y críticamente la información.

Específicamente, el investigador en su experiencia docente ha evidenciado en los estudiantes problemas para localizar de manera rápida datos e ideas en un texto, limitaciones para manifestar los objetivos de la lectura, dificultades al recuperar datos y estructurar acontecimientos del texto, contratiempos al analizar y argumentar el contenido e inconvenientes al jerarquizar la información para realizar predicciones y lanzar hipótesis sobre las lecturas.

Este tipo de dificultades e inconvenientes según Hoyos y Gallegos (2017), limitan la capacidad de comprender lo que se lee; complican el identificar el significado de las palabras que se incluyen en un texto; e impiden visualizarlo como un todo global, por lo que se dificulta elaborar una interpretación, así como reflexionar sobre su contenido. Lo anterior se traduce en bajos niveles de comprensión lectora y un restringido desarrollo de habilidades para alcanzarla, situaciones que a nivel general, afectan el desempeño académico en diversas áreas del saber.

De lo anterior, se derivó la necesidad de efectuar intervenciones a través de lecturas dinámicas e interactiva adjuntadas en el FB por ser la red social de mayor amplitud y usualmente manejada por los estudiantes desde edades tempranas (Piscitelli, Adaime y Binder, 2010). En este sentido, esta aplicación digital del internet y la web 2.0, puede propiciar cambios sustanciales a nivel de la comprensión lectora, en el rastreo e intercambio de información y análisis de datos textuales. Esta situación no solo permite 
el avance e innovación en el contexto académico y el desarrollo de estrategias de aprendizaje, sino también preparar cognoscitivamente a los estudiantes para enfrentar diversas situaciones que se les plantearán en la vida cotidiana.

Así la investigación que se describe a continuación, buscó determinar en un estudio de caso, la efectividad educativa del FB en el desarrollo de la comprensión lectora de un grupo de estudiantes del undécimo grado del Colegio Divino Niño de la Ciudad de Santa Marta, Colombia mediante la identificación de habilidades en esta área que, según la experiencia investigativa de autores tales como Hoyos y Gallegos (2017) se adquieren o consolidan por medio de un trabajo constante y estructurado en la sistematización de las funciones del pensamiento lector.

Este artículo está estructurado en cuatro partes: la primera muestra el fundamento teórico que soporta el estudio, la segunda el proceso metodológico utilizado para la recolección y procesamiento de los datos, la tercera describe los resultados obtenidos, siguiendo con la cuarta donde se presentan las conclusiones de la investigación.

\section{Marco teórico.}

La fundamentación teórica sobre la que se enmarca el estudio se ha dividido en tres subtemas: En primera instancia, se presenta un abordaje sobre la aplicación educativa del FB, seguido de la definición y habilidades de la comprensión lectora.

\section{Aplicación educativa de la red social Facebook.}

El Facebook es una de las herramientas inmersas en la Web 2.0, y más concretamente de las redes sociales representando no solo un medio más de comunicación, diálogo y colaboración, sino también como una herramienta de aprendizaje. Así, considerando el potencial educativo de las redes sociales, Garrigós et al., (2015), señala que son herramientas útiles para que un grupo de personas puedan potenciar la comunicación, sentirse parte de una comunidad y cooperar en tareas comunes. Estas características pueden hacer que su uso sea conveniente en entornos 
educativos, donde igualmente, es necesaria la interacción, colaboración e intercambio de información para el aprendizaje individual y grupal.

En este orden de ideas, Palmucci (2015), explica que la aplicación educativa del FB introduce cambios en la relación entre el docente-alumno, así como en la percepción del aula como espacio cerrado. El manejo de una red social con fines educativos, puede convirtiéndose en un nuevo espacio de diálogo e intercambios de saberes para dar lugar al aprendizaje colaborativo.

Asimismo agrega, que el FB proporciona un espacio virtual de socialización, cooperación y colaboración entre el alumnado al margen de la figura del profesor; es decir, un espacio de interacción que con un proyecto/ tarea bien definido por el docente, puede ser convertido en un espacio para el trabajo colaborativo de los alumnos y también fomentar su autonomía.

Para Piscitelli, Adaime y Binder (2010), la elección de esta red social se ha hecho por ser Facebook un medio masivo, de gran impacto en especial entre los jóvenes y que se ha convertido en una plataforma de fácil acceso para todas las personas. Por lo que su interfaz amigable e interactiva son algunas de sus grandes cualidades. Además, señala Nellison (2007), que Facebook ya está integrado en las prácticas diarias de los estudiantes y tiene el potencial de ayudar a perfeccionar sus habilidades de alfabetización digital, una habilidad cada vez más importante una vez que salen de las instituciones educativas a nivel superior.

Luego de analizar los diversos autores, Garrigós et al. (2015), Palmucci (2015), Piscitelli, Adaime y Binder (2010) y Nellison (2007), se observaron coincidencias en sus enunciados teóricos, por cuanto explicaron sobre todo en el aspecto colaborativo, donde enfatizan que a través de la red social FB tanto docentes como estudiantes, tendrán los espacios adecuados para compartir información y colaborar en la construcción de nuevos conocimientos. Con el tiempo el rol de red social del FB, se ha 
ido revaluando utilizándose en diferentes campos como lo es lo educativo, y por ello tanto docentes como estudiantes la están utilizando como una herramienta pedagógica.

\section{Comprensión Lectora.}

Desde la perspectiva de Araoz et al., (2014), la comprensión lectora implica un conjunto de habilidades desarrolladas a partir de la adquisición y ejercicio constante de estrategias tanto cognitivas como metacognitivas para generar nuevos conocimientos a partir del texto. Esto permite establecer conexiones entre el saber, vivencias y sentimientos del lector con lo captado del texto, pues por medio de la lectura se da un proceso interno de forma consciente o inconsciente en el cual se construyen nuevos significados a medida que aumenta el interés por el contenido.

En complemento a lo expuesto, Cerrillo y Cañamares (2017, p. 371), exponen que la comprensión lectora va "desde la identificación y recuerdo de un texto hasta su valoración crítica, pasando por la inferencia en sus variadas posibilidades, por medio de un proceso interactivo en el que el lector relaciona la información que posee con la que le brinda el texto". Lo define como un proceso donde el lector construye un significado al aplicar estrategias que lo hacen consciente de la comprensión del texto.

Se suma a lo anterior el aporte del Ministerio de Educación, Cultura y Deporte de España (MECD 2007, p.18), en el cual se expresa que la comprensión lectora es "la habilidad para comprender y utilizar las formas lingüísticas requeridas por la sociedad y/o valoradas por el individuo". Esto otorga a los lectores desde muy corta edad la capacidad de construir significado a una variedad de textos.

Para Pérez (2016, p.75) la comprensión lectora es el "proceso cognoscitivo por medio del cual se reconstruye en la mente del lector la información transmitida por el autor del texto, a través de un canal escrito, audiovisual o electrónico". Esto es, captar el significado de lo contenido en el texto conservando la información más general para integrarla a la nueva información y eliminando la secundaria. Por tanto, es un elemento facilitador del aprendizaje significativo y del alcance de competencias cognitivas. 
Basado en lo expuesto, los autores consultados coinciden en que la comprensión lectora es una actividad que involucra un proceso cognoscitivo para generar conocimiento a partir de la información que brindan los textos. Vale destacar lo expuesto por Araoz et al. (2014), relacionado con las conexiones dadas entre el saber, vivencias y sentimientos de la persona mientras lee, para finalmente, con lo que capta del texto incrementa su vocabulario, conocimientos y experiencias.

Por su parte, Cerrillo y Cañamares (2017), destacan que en la comprensión lectora prevalece la acción misma de leer y la valoración crítica dada por el lector una vez que establece inferencias al respecto. El MECD (2007), señala que las personas desde muy temprana edad desarrollan esta capacidad, por tanto viven esta experiencia temprana alimentando su capacidad lingüística. Mientras que Pérez (2016), engloba los aspectos semánticos y sintácticos que permiten al lector transformar y dar sentido a lo leído para convertirla en nueva información.

\section{Habilidades de Comprensión Lectora.}

Sin duda alguna la enseñanza de la lectura, es un aspecto primordial en el ser humano; de acuerdo con Gordillo y Flórez (2009), la comprensión lectora puede adquirirse a través de una serie de habilidades que permiten al lector extraer significado a un texto, así como activar los procesos perceptivos, lingüísticos y cognoscitivos. El sentido del texto se asegura al recurrir a esquemas para integrar coherentemente el contenido y emitir conclusiones propias.

Dentro de esas mismas ideas, los autores Gutiérrez y Salmerón (2012), señalan que la comprensión lectora es aquella que hace referencia a un proceso simultáneo de extracción y construcción transaccional entre las experiencias y conocimientos del lector, con el texto escrito en un contexto de actividad. Por su lado, el autor Molina (2007), expresa que la misma es un proceso complejo el cual incluye el uso consciente 
o inconsciente de varias estrategias, incluidas las de resolución de problemas, para reconstruir el significado que el autor ha querido comunicar.

Según Pérez (2016), las habilidades de la comprensión lectora se clasifican la siguiente forma: a) Comprensión literal: Implica identificar personajes, tiempos, espacios, enunciados textuales al igual que reconocer secuencias de significados y relaciones de semánticas causales, b) Reorganización de la información: Consiste en descomponer el texto en sus partes integrantes, con el objetivo de revelar su composición y estructura en elementos más simples, c) Comprensión inferencial: Con esta habilidad el lector logra interpretar el texto a partir de deducciones y relaciones del contenido con sus experiencias.

Por su parte Gordillo y Flórez (2009), asumen la comprensión lectora como producto de un conjunto de habilidades que debe desarrollar el estudiante para que pueda obtener un significado idóneo de una determinada lectura; para que de esta forma el mismo pueda emitir o crear un juicio propio sobre lo leído. Según, estos autores las habilidades de la comprensión lectora son las siguientes:

a) Comprensión literal: El lector reconoce las frases y las palabras claves del texto. Capta lo que el texto dice sin una intervención muy activa de la estructura cognoscitiva e intelectual del lector. Corresponde a una reconstrucción del texto que no ha de considerarse mecánica, comprende el reconocimiento de la estructura base del mismo. El lector con esta habilidad, fija su atención en las ideas principales que aparecen explicitas en éste y que se les hace fácil reconocer.

b) Reorganización de la información: Consiste en una reinterpretación personal de los símbolos verbales impresos que se justifica solo en la medida en que el lector es capaz de comprender los significados que están a su disposición. Esta habilidad permite al lector extraer las ideas concretas del texto, facilitando la interpretación general de este desde un punto de vista sistémico; de allí que pueda hacer categorizaciones, modelajes de situaciones, generar resúmenes, entre otras actividades. 
c) Comprensión inferencial: Con esta habilidad el lector puede ser capaz de combinar la información del texto con las experiencias previas para emitir conclusiones, hacer deducciones, contrastes de ideas, ayudado en sus experiencias vividas y en el bagaje de conocimientos que guarda de las dinámicas del entorno que lo rodea. Puede apreciar inferencias dentro del texto de: situaciones que no aparecen expresamente en él, puede por tanto presuponer y deducir lo implícito acerca de ideas principales, secundarias, atributos de personajes, entre otros aspectos.

d) Lectura crítica o juicio valorativo: Con esta habilidad el lector es capaz de emitir juicios sobre el texto leído aceptarlo o rechazarlo posturas con argumentos válidos. La lectura crítica tiene un carácter evaluativo, en el que interviene la formación del lector, su criterio y conocimientos de lo leído. Este nivel crítico tiene un carácter evaluativo, en el que interviene la formación del lector, su criterio y conocimientos sobre lo leído. e) Apreciación lectora: El lector realiza un análisis profundo del texto, con toda la objetividad y criticidad que requiere, dando apreciaciones relevantes sobre el mismo, mostrando un dominio de lo planteado en el texto, realizando inferencias de relaciones lógicas como: motivaciones, sucesos, causas de hechos; y de inferencias sobre relaciones de espacio o de tiempo.

Una vez comparados los aportes de los autores, se aprecia que en la clasificación de Pérez (2016) las habilidades de la comprensión lectora se agrupan en tres categorías: Comprensión literal, reorganización de la información y comprensión inferencial. Al respecto, Godillo y Flórez (2009) amplían tales habilidades incluyendo además de las antes mencionadas a la lectura crítica y apreciación lectora, describiendo en cada una de ellas los procesos cognitivos que conllevan a la comprensión e interpretación del texto escrito de forma significativa por tanto se tomó como referencia teórica la clasificación de estos autores. 


\section{Metodología.}

Siguiendo los aportes de Hurtado (2010), Hernández, Fernández y Baptista (2016) y Arias (2012), la investigación fue descriptiva, mientras que el diseño fue cuasiexperimental y transeccional-descriptivo. Por tratarse de un estudio de caso, basado en Méndez (2014) la muestra fue de tipo intencional constituida por los estudiantes del undécimo grado del Colegio El Divino Niño, de la ciudad de Santa Marta, Colombia que cumplieron los criterios de inclusión establecidos por el investigador, quedando integrada por cuarenta (40) educandos, subdivididos previo al estudio, en dos secciones de 20 alumnos cada una: A y B, considerando a la sección A como el grupo control y la B como el experimental.

Con respecto a la intervención didáctica diseñada para los grupos de estudio, ésta estuvo basada en la lectura de un texto de forma tradicional (impresos en papel) para el grupo control y en utilizar el Facebook por el grupo experimental para la lectura de textos colgados en la red, la misma fue implementada mediante una planificación de 20 horas durante las clases de Castellano y constó de 4 actividades, las cuales se describen brevemente:

a. Para la modalidad de lectura tradicional, los 20 estudiantes del grupo control se reunieron en el salón de clases para realizar la lectura con la utilización del texto escrito en papel.

b. Para la modalidad con la aplicación educativa de Facebook, los 20 estudiantes del grupo experimental se reunieron en el salón de clase de informática, se creó un grupo en Facebook, al cual se le llamó “Comprensión lectora en la red”. Seguidamente se les indujo a abrir el grupo creado anteriormente, procediendo leer el mismo texto del grupo control pero adjuntado en la red, donde se presentó el contenido escrito acompañado de imágenes planas y sonidos en el espacio virtual.

C. Luego de la lectura en ambos grupos el docente facilitó la dinámica adaptada a cada ambiente de aprendizaje para extraer las ideas reflexivas, críticas e interpretativas sobre el contenido del texto. En el grupo control consistió en responder un 
cuestionario escrito sobre la lectura y en el experimental los estudiantes participaron en el grupo de FB creado para compartir ideas sobre la lectura realizada. En ambos casos el docente orientó la dinámica para promover las habilidades de la comprensión lectora: comprensión literal, comprensión inferencial, reorganización de la información, lectura crítica o juicio valorativo y apreciación lectora.

d. Acto seguido, se realizó una comparación entre los estudiantes que recibieron clases de forma tradicional con los que recibieron clase con la aplicación educativa Facebook desde dos puntos de vista, el primero fue observando la motivación de los estudiantes para hacer la actividad desde la red social fue más entusiasta que la clase recibida de manera magistral y rutinaria, por lo tanto, los avances fueron más significativos, ya que fue evidente un mayor interés para desarrollar el trabajo a través de la red social. El segundo, correspondió a la comparación estadística de los resultados obtenidos de la prueba de conocimiento aplicada a ambos grupos para demostrar la hipótesis de la investigación.

Una vez realizada la intervención se procedió a la recolección de los datos que permitieron demostrar la efectividad de la aplicación educativa del FB para el desarrollo de la compresión lectora. En este orden de ideas, el investigador sustentado en Hurtado (2010), utilizó la técnica de la encuesta, y como instrumento, una prueba de conocimiento.

La estructura la prueba estuvo conformada por 15 ítems para evaluar las habilidades de la comprensión lectora antes mencionadas. Cabe destacar que dicha prueba por constituir el instrumento de recolección fue validado de acuerdo a lo indicado por Hernández, Fernández y Baptista (2016) y utilizado en una prueba piloto para calcular la confiabilidad a través del coeficiente K-20 de Kuder-Richardson, el cual fue de 0,868 , indicando muy alta confiabilidad según la escala propuesta por Palella y Martins (2011). 
Una vez determinada la validez y confiabilidad, la prueba de conocimiento se consideró apta para ser aplicada en los grupos control (A) y experimental (B), para medir la comprensión lectora post intervención. De forma que, los participantes debieron responder esta serie de preguntas o situaciones adecuadamente definidas en forma y contenido.

Con respecto a la técnica de análisis de datos, se procedió de acuerdo con en Hernández et al., (2016), a calcular las medias aritméticas de los indicadores de la variable comprensión lectora: Lectura literal, reorganización de la información, inferencial, lectura crítica y apreciación lectora; en los dos control y experimental. En este caso se realizó un baremo para el análisis de las medias calculadas aportando la apreciación del nivel alcanzado para las habilidades y comprensión lectora evaluadas. (Ver cuadro 1)

\section{Cuadro 1}

Baremo para el análisis de las medias

\begin{tabular}{|c|c|}
\hline INTERVALO & CATEGORÍA \\
\hline$(0-0,69)$ & Muy bajo \\
\hline$(0,7-1,29)$ & Bajo \\
\hline$(1,3-1,79)$ & Medio \\
\hline$(1,8-2,49)$ & Alto \\
\hline$(2,5-3)$ & Muy alto \\
\hline
\end{tabular}

Fuente: Elaboración propia

La efectividad del educativa FB se determinó a través de la comparación estadística mediante el cálculo de la $\mathrm{T}$ de Student de los promedios de obtenidos por ambos grupos en la prueba de conocimiento, teniendo como hipótesis de investigación que el desarrollo de la comprensión lectora en los estudiantes que utilizaron el FB es significativamente superior a la obtenida por los estudiantes que aplicaron el método de lectura tradicional. 


\section{Resultados.}

La efectividad de la aplicación educativa del FB para la compresión lectora fue determinada a través de la prueba $\mathrm{T}$ de Student asumida como un estadístico comparativo de las medias aritméticas obtenida en la prueba de conocimiento por los estudiantes que efectuaron la lectura del texto en forma tradicional con los que lo hicieron a través del FB, teniendo como resultados los puntajes que se indican a continuación en la tabla 1 para las habilidades de la compresión lectora evaluadas como indicadores de la variable dependiente.

Tabla 1

Habilidades de la comprensión lectora Grupo control vs. Grupo experimental

Postest

\begin{tabular}{|l|c|c|c|c|}
\hline \multirow{2}{*}{$\begin{array}{c}\text { Indicadores: } \\
\text { Habilidades de la } \\
\text { comprensión lectora }\end{array}$} & \multicolumn{2}{|c|}{ Grupo Control } & \multicolumn{2}{c|}{ Grupo Experimental } \\
\cline { 2 - 5 } & Promedio & Categoría & Promedio & Categoría \\
\hline Comprensión literal & 3,00 & Muy alto & 3,00 & Muy alto \\
\hline $\begin{array}{l}\text { Reorganización de la } \\
\text { información }\end{array}$ & 1,70 & Medio & 2,10 & Alto \\
\hline Comprensión inferencial & 1,70 & Medio & 1,95 & Alto \\
\hline Lectura crítica & 0,95 & Bajo & 2,65 & Muy alto \\
\hline Apreciación lectora & 0,95 & Bajo & 1,85 & Alto \\
\hline Comprensión Lectora & $\mathbf{1 , 6 6}$ & Medio & $\mathbf{2 , 3 1}$ & Alto \\
\hline
\end{tabular}

Fuente: Elaboración propia

En la tabla 1, se observan diferencias entre los promedios alcanzados por los grupos para cada uno de los indicadores evaluados, excepto en comprensión literal, en el cual ambos grupos obtuvieron iguales promedios (3.00). Se observa que, en el resto de ellos, el grupo experimental demuestra un mayor desarrollo de las habilidades de la comprensión lectora. 
Asimismo, los promedios y categorías para la dimensión total, evidencian que los estudiantes del grupo control obtuvieron 1,66 puntos, categoría Medio, y los del grupo experimental obtuvieron 2,31 categoría Alto. Estas diferencias fueron corroboradas mediante la aplicación del estadístico t de Student, para grupos independientes, cuyos resultados se muestran en la tabla 2.

Tabla 2

Prueba $t$ de Student

Grupo control vs. Grupo experimental

Postest

\begin{tabular}{|l|c|c|}
\hline & $\begin{array}{l}\text { Grupo } \\
\text { control }\end{array}$ & $\begin{array}{l}\text { Grupo } \\
\text { Experimental }\end{array}$ \\
\hline$\Sigma$ & 8.3 & 11.55 \\
\hline Media & 1.66 & 2.31 \\
\hline Observaciones & 20 & 20 \\
\hline Diferencia hipotética de las medias & \multicolumn{2}{|c|}{0} \\
\hline Grados de libertad & \multicolumn{2}{|c|}{11,77} \\
\hline Estadístico t & \multicolumn{2}{|c|}{1,68} \\
\hline Valor crítico de t (una cola) & \multicolumn{2}{|c|}{0,05} \\
\hline Nivel de confianza & \multicolumn{2}{|c|}{} \\
\hline
\end{tabular}

Fuente: Elaboración propia

En la tabla 2, se observa que el valor calculado de $\mathrm{t}$ es mayor que su valor crítico establecido en las tablas estadísticas, lo cual, según Hurtado (2010) se interpreta como la existencia de diferencias significativas entre los grupos. En este caso se asumió de acuerdo con Hernández et al (2016), el supuesto establecido para la prueba t de Student de dos muestras independientes que contempla una diferencia hipotética entre las medias es cero (0), es decir se presume que son iguales para comprobar a través del estadístico si existen o no diferencias entre ellas.

Como puede apreciarse la t calculada resultó igual a 11,77 mientras que el valor crítico de $t$ en las tablas es de 1,68 a un nivel de confianza de 0,05. Puede decirse 
entonces, que existe diferencia estadísticamente significativa entre las medias comparadas en cuanto al desarrollo de la comprensión lectora de los grupos en estudio a favor del grupo experimental, lo cual puede adjudicarse a su participación en las actividades mediadas por el aplicativo Facebook.

Con base en estos resultados, se evidencia la efectividad educativa del FB para el desarrollo de la comprensión lectora de los estudiantes del undécimo grado del Colegio El Divino Niño, de la ciudad de Santa Marta, Colombia.

\section{Discusión.}

Los resultados tras el uso de la aplicación Facebook en el grupo experimental, son claramente diferenciados del grupo control, en tal sentido, las valoraciones cualitativas de las habilidades de comprensión lectora del grupo experimental, se corresponden al nivel de desarrollo muy alto y alto.

Tomando como referencia el resultado de las medias las aritméticas de las habilidades de la comprensión lectora, éstas fueron superiores en el grupo experimental. Al respecto, se observó en el indicador compresión literal, correspondencia con Gordillo y Flórez (2009), al plantear que en este nivel, el lector reconoce las frases y palabras claves del texto sin una intervención muy activa de su estructura cognoscitiva e intelectual.

Asimismo, en la compresión literal, el lector fija su atención en las ideas principales que aparecen explícitas en el texto y que se les hace fácil reconocer. Este resultado demuestra, que a los estudiantes luego de utilizar la aplicación Facebook, se les facilitó el nivel de lectura básico, realizando una exploración inicial del texto teniendo en cuenta los aspectos fundamentales del tema.

Por otra parte, en relación al indicador reorganización de la información, se aprecia coincidencia con lo expuesto por Pérez (2016), en relación a que en este nivel el lector extrae las ideas concretas del texto para una comprensión general de su contenido, desde 
un punto de vista sistémico, de allí que pueda hacer categorizaciones, modelajes de situaciones, generar resúmenes, entre otras actividades interpretativas.

De igual forma, en relación al indicador comprensión inferencial, se observa correspondencia con Gordillo y Flórez (2009), al explicar que este nivel se caracteriza por dar cuenta de relaciones y asociaciones de significados que permiten al lector leer entre líneas, presuponer y deducir lo implícito; es decir, puede dar una explicación amplia del texto, adicionarle datos nuevos; algo muy importante, hacer intertextualidad con conocimientos ya adquiridos, lo que le permitirá sacar sus propias conclusiones, generar hipótesis y todavía más, aportar ideas innovadoras. Este nivel de comprensión requiere del lector un considerable grado de abstracción. Favorece la relación con otros campos del saber y la integración de nuevos conocimientos en un todo.

Con respecto al indicador lectura crítica, se observó similitud con el planteamiento de Gordillo y Flórez (2009), en el que consideran a este nivel de comprensión lectora como el ideal, ya que el lector es capaz de emitir juicios sobre el texto leído, aceptarlo o rechazarlo, pero con argumentos. La lectura crítica tiene un carácter evaluativo, en el que interviene la formación del lector, su criterio y conocimientos e interpretación sobre lo leído.

Por su parte, el comportamiento del indicador apreciación lectora, muestra relación con Gordillo y Flórez (2009), al explicar que en dicha apreciación, el lector realiza un análisis profundo del texto, con toda la objetividad y criticidad que se requiere, dando apreciaciones relevantes sobre el mismo, mostrando un dominio de lo planteado en el texto, realizando inferencias sobre sucesos, causa de hecho, así como sobre ambigüedades y abstracciones.

Adicionalmente, el muy alto nivel de comprensión lectora alcanzado por los estudiantes luego de utilizar el Facebook, indica según Gordillo y Flórez (2009), que lograron dominar el proceso de extracción y construcción transaccional entre las experiencias y conocimientos del lector, con el texto escrito en una trama específica. 
En forma general, la efectividad del Facebook para el desarrollo de la comprensión lectora, es un resultado que se corresponde con los de Mora, Párraga, y Vargas (2014) quienes concluyeron que el uso del Facebook facilita la apropiación de procesos de la lectura en los estudiantes. Asimismo, se relaciona con Espuny, González, Lleixá y Gisbert (2011), quien considera la red social Facebook como la más utilizada, además debido a su popularidad y fácil acceso tanto para docentes como estudiantes, quienes la pueden utilizar sin ninguna restricción como una herramienta de apoyo pedagógico.

\section{Consideraciones finales.}

Los estudiantes que recibieron clases tradicionales, obtuvieron un nivel medio de comprensión lectora en el postest. Para este grupo, la comprensión literal se ubicó en un nivel muy alto, sin embargo, en las habilidades más complejas, tales como la reorganización de la información y la comprensión inferencial, obtuvieron un nivel medio, así como un nivel bajo en lectura crítica y apreciación lectora.

Los estudiantes que interactuaron con el FB, obtuvieron en el postest un nivel alto de compresión lectora. Éstos reforzaron la lectura literal, manteniéndose en un nivel muy alto, y se consolidaron todas las habilidades lectoras en niveles altos y muy altos.

Los grupos demostraron ser diferentes luego de la participación del grupo experimental en la intervención mediada por el Facebook en cuanto a los niveles de comprensión lectora. El grupo control demostró un nivel medio, mientras que el experimental alcanzó un nivel muy alto. Esta diferencia es estadísticamente significativa a favor del grupo experimental, demostrándose la efectividad educativa del Facebook para el desarrollo de la compresión lectora.

Basado en los hallazgos de la investigación, se recomienda:

a. Diagnosticar periódicamente la comprensión lectora de los estudiantes de la institución, a fin de identificar cuáles son sus fortalezas y debilidades para reforzar las primeras y disminuir las segundas. Del mismo modo, se sugiere llevar un registro 
sistemático acerca de los progresos de los estudiantes en esta área básica de su formación.

b. Monitorear la cuenta de Facebook creada para verificar los avances de los estudiantes en el desarrollo de su comprensión lectora.

C. Implementar las actividades realizadas mediante el aplicativo Facebook con la finalidad de desarrollar la comprensión lectora de los estudiantes, ya que la misma demostró ser efectiva para este propósito. Asimismo, se debe considerar incluir las modificaciones pertinentes para mantener la cuenta activa, suscribiendo nuevos miembros y ampliando las actividades para el logro de los objetivos planteados.

d. Diseñar una estrategia de intervención a través del Facebook dirigida a los participantes del grupo control, para que tengan la oportunidad de desarrollar habilidades tal como el grupo experimental. En este sentido, podrían incorporarse al grupo ya creado, para compartir experiencias y promover el intercambio entre pares.

e. Continuar reforzando las habilidades ganadas en cuanto a la comprensión lectora de los estudiantes, así como promover actividades docentes tales como un grupo virtual de discusión de lecturas interactivas, para mantenerlos motivados hacia la lectura.

f. Realizar investigaciones futuras donde se incluyan a los docentes en los grupos de estudio para indagar sobre sus competencias para adoptar la herramienta del FB y evaluar la efectividad obtenida. Además, es pertinente plantear el estudio de los lineamientos institucionales que apoyan las iniciativas de apropiación de las redes sociales para innovar en el proceso enseñanza-aprendizaje.

\section{Referencias Bibliográficas.}

Arias, Fidias (2012). El proyecto de investigación: Introducción a la metodología científica. (Sexta edición). Editorial Episteme. Venezuela.

Araoz, María Edith; Guerrero, Patricia; Galindo, María de los Ángeles; Villaseñor, Rosa y De La Vara, Ana (2014) Estrategias para aprender a aprender: 
reconstrucción del conocimiento a partir de la lectoescritura. (Segunda edición). Ediciones Prentice Hall. México.

Cerrillo, Pedro y Cañamares, Cristina. (2017) Literatura infantil: Nuevas lecturas y nuevos lectores. Ediciones de la Universidad de Castilla-La Mancha. España.

Nellison, Neil (2007). Facebook as a teaching tool? Recuperado de: http://nellison.blogspot.com/2007/12/ecar-facebook-as-teaching-tool.html Consulta: $12 / 02 / 17$

Espuny, Cinta; González, Juan; Lleixà, Mar y Gisbert, Merce. (2011). Actitudes y expectativas del uso educativo de las redes sociales en los alumnos universitarios. Revista de Universidad y Sociedad del Conocimiento. Volumen 8, No. 1. España. (Pp171-185).

Garrigós, Irene; Mazón, José; Saquete, Estela; Puchol, Marcel y Moreda, Paloma (2015). La influencia de las redes sociales en el aprendizaje colaborativo. Jornadas de Enseñanza Universitaria de la Informática, Santiago de Compostela. Recuperado de https://upcommons.upc.edu/bitstream/handle/2099/11859/p67.pdf Consulta: $16 / 02 / 17$

Gordillo, Adriana y Flórez, María del Pilar (2009) Los niveles de comprensión lectora: hacia una enunciación investigativa y reflexiva para mejorar la comprensión lectora en estudiantes universitarios. Revista Actualidades Pedagógicas. No. 53. México. (Pp. 95-107)

Guthrie, John y Taboada, Ana (2005). La lectura en materia de contenido: lo que sabemos y lo que necesitamos saber. Lectura y vida: Revista latinoamericana de lectura, Volumen 26, No. 1, Argentina... (Pp. 6-13).

Gutiérrez, Calixto y Salmerón Honorio (2012) Estrategias de comprensión lectora: enseñanza y evaluación en educación primaria. Recuperado de https://www.ugr.es/ recfpro/rev161ART11.pdf. Consulta: 15/02/17

Hernández, Roberto, Fernández, Carlos, y Batista, Pilar (2016). Metodología de la Investigación. (Quinta edición). Editorial McGraw Hill. México

Hoyos, Ana María y Gallego, Teresita (2017). Desarrollo de habilidades de comprensión lectora en niños y niñas de la básica primaria. Recuperado de http://revistavirtual.ucn.edu.co/index.php/RevistaUCN/article/view/841/1359 Consulta: 20/01/18

Hurtado, Jacqueline (2010). Metodología de la Investigación. Guía para la comprensión holística de la ciencia. (Cuarta edición). Editorial Quirón. Venezuela.

Méndez, Carlos (2014) Metodología. (Cuarta edición). Editorial Limusa, Grupo Noriega Editores. México 
Ministerio de Educación, Cultura y Deporte (2007) Estudio Internacional de Progreso en Comprensión Lectora de la IEA. PIRLS 2006. Informe español. Catálogo de publicaciones del MECD. España.

Molina, María del Rosario (2007) El profesor de audición y lenguaje ante el nuevo milenio. Editorial ESIC. España

Mora, Leydi; Párraga, Singrid y Vargas, Álvaro (2014) Facebook: una estrategia digital para desarrollar la producción y comprensión textual del género narrativo. Recuperado de: https://repository.uniminuto.edu/bitstream/handle/10656/2880/THUM_Mora BenavidesLeidyJohana_2013.pdf?sequence $=1$. Consulta: 20/03/17

Palella, Santa y Martins, Filiberto (2011) Metodología de la Investigación Cuantitativa. Ediciones FEDUPEL. Venezuela

Palmucci, María Fernanda (2015). El docente en la escena del aula y el uso de las TIC en su estrategia educativa. Recuperado de: http://fido.palermo.edu/servicios_dyc/publicacionesdc/vista/detalle_articulo.p hp?id_articulo=11010\&id_libro=535. Consulta: 21/02/17

Pérez, María Jesús (2016). Evaluación de la comprensión lectora: Dificultades y limitaciones. Revista de Educación. Número extraordinario. España. (Pp. 121-138)

Piscitelli, Alejandro; Adaime, Iván y Binder, Inés (2010). El proyecto Facebook y la posuniversidad. Sistemas operativos sociales y entornos abiertos de aprendizaje. Recuperado de: http://www.codajic.org/sites/www.codajic.org/files/El\%20Proyecto\%20Face book.pdf. Consulta: 30/05/17 